\title{
Pilot studies demonstrate the potential benefits of antiinflammatory therapy in human lymphedema
}

\author{
Stanley G. Rockson, ${ }^{1}$ Wen Tian, ${ }^{2}$ Xinguo Jiang, ${ }^{2}$ Tatiana Kuznetsova, ${ }^{3}$ Francois Haddad, ${ }^{1}$ Jamie \\ Zampell, ${ }^{4}$ Babak Mehrara, ${ }^{4}$ Joshua P. Sampson, ${ }^{1}$ Leslie Roche, ${ }^{1}$ Jinah Kim, ${ }^{5}$ and Mark R. Nicolls ${ }^{2}$ \\ 'Department of Medicine, Stanford University School of Medicine, Stanford, California, USA. ²Department of Medicine, \\ VA Palo Alto Health Care System/Stanford University, Palo Alto, California, USA. ${ }^{3}$ Research Unit Hypertension and \\ Cardiovascular Epidemiology, KU Leuven Department of Cardiovascular Sciences, University of Leuven, Belgium. \\ ${ }^{4}$ Department of Surgery, Memorial Sloan Kettering Cancer Center, New York City, New York, USA. ${ }^{5}$ Department of \\ Pathology, Stanford University School of Medicine, Stanford, California, USA.
}

BACKGROUND. Lymphedema is a common condition affecting millions around the world that still lacks approved medical therapy. Because ketoprofen, an NSAID, has been therapeutic in experimental lymphedema, we evaluated its efficacy in humans.

METHODS. We first performed an exploratory open-label trial. Patients with either primary or secondary lymphedema received ketoprofen $75 \mathrm{mg}$ by mouth 3 times daily for $\mathbf{4}$ months. Subjects were evaluated for changes in histopathology, with skin thickness, limb volume, and tissue bioimpedance changes serving as secondary endpoints. Based on our encouraging findings, we next conducted a placebo-controlled trial, with the primary outcome defined as a change in skin thickness, as measured by skin calipers. Secondary endpoints for this second study included histopathology, limb volume, bioimpedance, and systemic inflammatory mediators.

RESULTS. We enrolled 21 lymphedema patients in the open-label trial, from November 2010 to July 2011. Histopathology and skin thickness were significantly improved at 4 months compared with baseline. In the follow-up, double-blind, placebo-controlled trial, we enrolled 34 patients from August 2011 to October 2015, with 16 ketoprofen recipients and 18 placebo-treated subjects. No serious adverse events occurred. The ketoprofen recipients demonstrated reduced skin thickness, as well as improved composite measures of histopathology and decreased plasma granulocyte CSF (G-CSF) expression.

CONCLUSION. These 2 exploratory studies together support the utility of targeted antiinflammatory therapy with ketoprofen in patients with lymphedema. Our results highlight the promise of such approaches to help restore a failing Iymphatic circulation.

TRIAL REGISTRATION. ClinicalTrials.gov NCT02257970.

Conflict of interest: MRN is a consultant and share-holder for Eiger Biopharmaceuticals, a company evaluating LTB4 antagonism as a treatment for lymphedema.

License: Copyright 2018, American Society for Clinical Investigation.

Submitted: July 24, 2018 Accepted: September 6, 2018 Published: October 18, 2018

Reference information: JCI Insight. 2018;3(20):e123775. https://doi.org/10.1172/jici. insight.123775.

\section{Introduction}

Lymphedema occurs when impaired fluid transport through the lymphatic circulation leads to nonresolving, progressively nonpitting fluid retention and can be broadly divided into primary and secondary forms (1). Primary lymphedema is due to inherent abnormalities of the lymphatic circulation (2), whereas secondary forms of the disease typically occur when a new obstruction occurs, such as with lymphatic filariasis (3) or following lymph node resection (4). The latter form of disease is the most common manifestation and affects as many as 200 million patients worldwide and at least 3 million in the US (5). There are significant unmet medical needs for this large and underserved patient population (6); drug therapy that can safely and effectively treat this chronic, debilitating condition is not available.

Lymphedema ensues as a consequence of relative lymphatic vascular insufficiency (2). The disease is characterized by an imbalance of growth factors $(7,8)$ and by the presence of persistent tissue inflammation (1). While therapeutic approaches that foster reparative lymphangiogenesis might, in the future, have the 
potential to reverse the pathology of lymphedema $(9,10)$, the relationship between persistent inflammation and impaired lymphangiogenesis has not, until recently, been sufficiently explored $(11,12)$.

It is postulated that chronic interstitial fluid accumulation in lymphedema leads both to the architectural changes in the structure of the skin and to impaired clearance of inflammatory cells and mediators. In order to explore the molecular pathogenesis of this disease, we have previously investigated a murine model of acquired, postsurgical lymphedema (13). In this prior study, the presence of intense histopathological inflammatory changes in the dermis correlated with impaired mobilization of immunocompetent cells from the lymphedematous regions. Large-scale, transcriptional profiling of the lymphedematous tissues disclosed a distinct, predominant inflammatory molecular expression profile that led us to postulate a role for leukotrienes, both in the pathogenesis of the disease and as a potential therapeutic target (13). With these results, and the unresolved need for drug therapy, we decided to investigate the therapeutic impact of ketoprofen (14), an NSAID agent with a recognized dual antiinflammatory mechanism of action that includes inhibition of the 5-lipoxygenase $(5$-LO) pathway $(15,16)$. When ketoprofen was systemically administered to mice with experimental, acquired lymphedema, there was documented reversal of disease burden, including remarkable normalization of the pretreatment lymphedematous histopathology (14). Our subsequent preclinical investigations, both in vitro and in vivo (17), strongly suggest that the therapeutic benefit of ketoprofen in experimental lymphedema is specifically attributable to its inhibition of the 5-LO pathway. In light of these preclinical insights, we designed the current investigations to evaluate the potential for ketoprofen to therapeutically ameliorate the morbidity of human lymphedema, a disease for which there is no currently available pharmacotherapy.

\section{Results}

Study design and participants for open-label and placebo-controlled trials. These studies were conducted at a single academic site (Stanford University). We first performed an exploratory open-label trial. Human subjects with primary or secondary lymphedema of either upper or lower extremity were considered for this trial. Based on the results of this preliminary study, we conducted a double-blind, placebo-controlled, single-center trial (Stanford University School of Medicine). The enrollment schema for the sequential investigations is presented in Figure 1.

Inclusion criteria for open-label and placebo-controlled trials. Participants, aged 18-90 years, with a history of lymphedema in one or more limbs (unilateral or bilateral) of $>6$ months' duration were considered. To be eligible for screening with unilateral disease, the patient was required to demonstrate a $\geq 20 \%$ volume increment when compared with the contralateral, normal limb; in the context of bilateral disease, the physical findings of lymphedema, coupled with the appropriate risk profile, sufficed. In all cases, the classic skin changes of lymphedema were required. A subject was eligible after completing a course of decongestive physiotherapy with subsequent consistent use of prescribed compression garments.

Exclusion criteria for open-label and placebo-controlled trials. Patients with established cardiovascular disease, diabetes, hypertension, or tobacco use were excluded from the trials. Patients with active cancer, current infection, or a bleeding diathesis were ineligible. Additional exclusion criteria included relative or absolute contraindication to NSAID use, including a history of allergies, known gastrointestinal intolerance, or other life-threatening or progressive systemic illnesses (e.g., renal failure, hepatic dysfunction, congestive heart failure, or neurological or psychological impairment) that would impair the patients' ability to participate.

Patient characteristics for open-label and placebo-controlled trials. In total, 23 patients were screened for the openlabel trial. We enrolled 21 patients, with 16 (76\%) completing the open-label study. The subsequent placebocontrolled trial screened 44 patients and enrolled 34 patients, with $30(88 \%)$ completing this study. The clinical characteristics of patients who completed the open-label and placebo-controlled trials are presented in Table 1. For the placebo-controlled trial, there were no significant differences among ketoprofen- and placebo-treated subjects with respect to age, race, duration of lymphedema, histopathology score, skin thickness, limbs affected, lymphedema etiology (primary vs. secondary), or cause of secondary disease (cancer- vs. non-cancer-related). For the placebo group, the noncancer causes of secondary lymphedema were a consequence of massive infection, trauma, and noncancer surgery on the left knee. For the ketoprofen group, the noncancer causes of secondary lymphedema were noncancer abdominal surgery, morbid obesity, and May-Thurner syndrome.

Treatment and outcome measures for open-label and placebo-controlled trials. Given limited clinical precedents, we empirically chose a histological endpoint for the open-label trial based on our prior investigation of ketoprofen responsiveness in experimental lymphedema (14). To evaluate the impact of ketoprofen treatment 


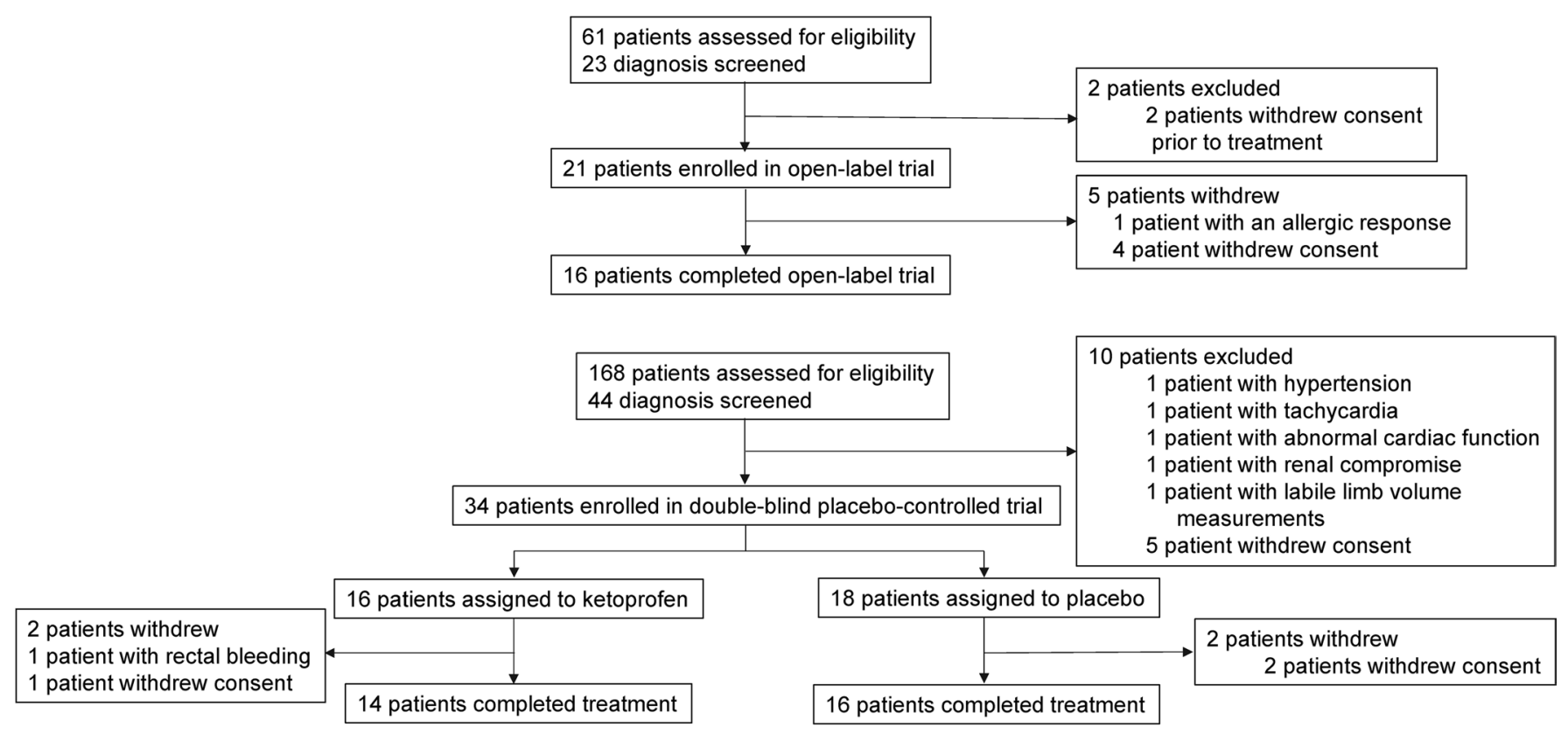

Figure 1. Overview of patient flow and disposition in the open-label and placebo-controlled trials.

on human cutaneous histopathology, we created a dermatopathological scoring system that incorporates dermal thickness, intercellular mucin content, deep dermal collagen content, and perivascular infiltrate; this quantitative assessment was developed and performed by a dermatopathologist (J. Kim; see Methods for details). The histopathological scoring system was derived from the prospective analysis of a training set composed of equal numbers of cutaneous biopsies derived from normal and lymphedematous skin. For a clinical correlate to the disease-related changes in cutaneous architecture, we concurrently evaluated changes in skin thickness as measured by skin calipers. In addition, we measured limb volume and tissue bioimpedance spectroscopy (as an indirect measure of extracellular fluid content). To establish parallels with the salient findings in the animal model, we chose to evaluate therapeutic amelioration of the histopathology in the skin, along with its clinical counterpart, changes in caliper-measured skin thickness. Because of the findings of the open-label study, we elected to use skin thickness as a clinically relevant primary endpoint in the lymphedema response to therapeutic intervention; for this reason, in the placebo-controlled trial, we changed the primary outcome measure to skin thickness. The secondary endpoints for this latter study included changes in cutaneous histopathology, alterations in limb volume, and an assessment of circulating, systemic inflammatory mediators. In the open-label study, patients received $75 \mathrm{mg}$ ketoprofen by mouth 3 times a day for 4 months; for the placebo-controlled trial, either the same ketoprofen dose or its placebo was administered for the same duration.

Ketoprofen ameliorates skin pathology in human lymphedema. The open-label trial showed that ketoprofen treatment produced a marked improvement in the mean histopathology score compared with baseline (post-minus prescores, $-3.4 \pm 0.6, P<0.0001$; Figure $2 \mathrm{~A}$ ). Additionally, ketoprofen treatment led to a significant reduction in skin thickness $(62.1 \pm 8.4 \mathrm{~mm}$ before treatment vs. $27.4 \pm 5.6 \mathrm{~mm}$ after treatment, $P=0.0006$; Figure 2B). There was no significant difference in either limb volume or bioimpedance when baseline values were compared with those at study termination (data not shown).

We confirmed the effect of ketoprofen on the predefined primary outcome measure of skin thickness in the placebo-controlled trial. The mean skin thickness in the ketoprofen-treated patients was significantly reduced from $49.4 \pm 5.7 \mathrm{~mm}$ before treatment to $41.4 \pm 5.8 \mathrm{~mm}$ after treatment $(P=0.01)$, while placebo-treatment did not result in a significant change ( $49.7 \pm 7.0 \mathrm{~mm}$ before treatment to $47.4 \pm 7.7 \mathrm{~mm}$ after treatment; Figure $3 \mathrm{~A})$. The change in the histopathology score was significantly better in the ketoprofen group than the placebo group $(-3.0 \pm 0.5$ before treatment vs. $1 \pm 0.5$ after treatment, $P=0.03$; Figure $3 \mathrm{~B})$. There were no significant differences in microlymphatic vascular area in the skin when ketoprofen specimens were compared with placebo (results not shown); however, changes in vascular area correlated directly with the changes in the histopathology score $(r=0.6, P<0.05$, Supplemental Figure 1; supplemental material available online with this article; 
Table 1. Baseline clinical characteristics of all treated patients

\begin{tabular}{|c|c|c|c|}
\hline Age (years) & $57 \pm 10$ & $55 \pm 13^{A}$ & $43 \pm 16$ \\
\hline Histopathological score ${ }^{c}$ & $6.1 \pm 0.6$ & $2.7 \pm 0.5^{A}$ & $4.1 \pm 0.4$ \\
\hline Caliper skin thickness (mm) & $62 \pm 8.4$ & $50 \pm 7.0^{\mathrm{A}}$ & $49 \pm 5.7$ \\
\hline Female sex - no. (\%) & $13(81)$ & $14(100)^{A}$ & $13(87)$ \\
\hline Black - no. (\%) & $0(0)$ & $0(0)^{B}$ & $0(0)$ \\
\hline Asian - no. (\%) & $1(7)$ & $1(7)^{\mathrm{B}}$ & $0(0)$ \\
\hline Other - no. (\%) & $1(7)$ & $1(7)^{\mathrm{B}}$ & $2(13)$ \\
\hline Duration of Lymphedema - years & $12 \pm 7$ & $11 \pm 9^{B}$ & $7 \pm 6$ \\
\hline \multicolumn{4}{|l|}{ Limb(s) Affected } \\
\hline Primary - no. (\%) & $5(31)$ & $1(7)^{\mathrm{B}}$ & $4(25)$ \\
\hline Secondary - no. (\%) & $11(69)$ & $13(93)^{B}$ & $12(75)$ \\
\hline \multicolumn{4}{|l|}{ Secondary Lymphedema } \\
\hline Cancer-related - no. (\%) & $8(73)$ & $6(46)^{\mathrm{B}}$ & $8(67)$ \\
\hline
\end{tabular}

${ }^{A}$ Ketoprofen-to-placebo comparison not significant, unpaired $t$ test. ${ }^{B}$ Ketoprofen-to-placebo comparison not significant, Fisher exact test.

'Histopathological Score defined in Methods. ${ }^{D}$ All upper extremity cases were unilateral

https://doi.org/10.1172/jci.insight.123775DS1). As in the open-label study, the placebo-controlled trial did not demonstrate a reduction of limb volume or bioimpedance (Table 2). In the placebo-controlled study, we also compared the systemic inflammatory responses (longitudinal analysis) of the 2 treatment groups, using a 62-plex Luminex-bead analysis (Supplemental Table 1) of pre- and posttreatment plasma samples (Table 3). In order to account for interaction among the assayed biomarkers, a partial least squares (PLS) analysis was undertaken, depicted graphically as volcano plots for both absolute (Supplemental Figure 2) and relative (Supplemental Figure 3) changes. Significance for individual cytokines was defined by a variables importance for projection (VIP) $>1.5$. Using this approach, granulocyte CSF (G-CSF) was significantly decreased by ketoprofen treatment when compared with placebo, both in terms of absolute and relative change.

Cellulitis is a recognized form of morbidity that is commonly encountered in lymphedema patients. Although there is a relationship between immune status and the risk of cellulitis, this study was not powered to detect an effect on cellulitis incidence. Six placebo-treated lymphedema patients had a history of cellulitis, but cellulitis did not occur in any patients during the study period. In the ketoprofen group, 1 person had a history of cellulitis approximately 1 year before entering the study, but no patients developed cellulitis during the study period.

There were no serious adverse events in these trials. Gastrointestinal effects are known to limit the effects of NSAIDs. In this study, in the placebo-controlled component, 1 ketoprofen-treated patient, with a prior history of hemorrhoids, experienced rectal bleeding and withdrew. A second ketoprofen-treated patient developed nonhemorrhagic dyspepsia but was able to complete the trial.

Ketoprofen is a unique NSAID that possesses dual pathways of inflammatory inhibition, blocking both cyclooxygenase (COX) and 5-LO. Our group recently determined that ketoprofen's mechanism of action in experimental lymphedema is mediated by its negative effect on leukotriene B4 ( $\left.\mathrm{LTB}_{4}\right)$ production, via inhibition of 5-LO. For this reason, we evaluated the impact of ketoprofen treatment on immunohistochemical 5-LO tissue expression in cutaneous biopsies of the enrolled patients, documenting that there was a significant ketoprofen-induced reduction in 5-LO-specific staining in tissue biopsies that was not observed in the placebo recipients (Figure 4). 
A Open-Label Trial $1^{\circ}$ Endpoint: Histology

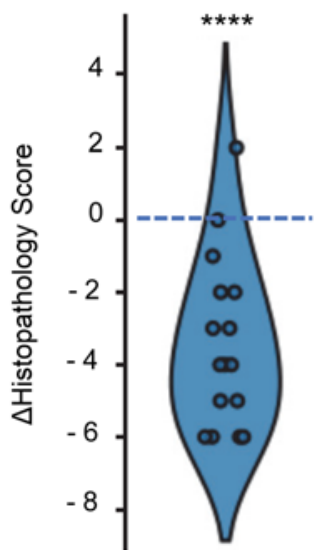

B Open-Label Trial $2^{\circ}$ Endpoint: Skin Thickness

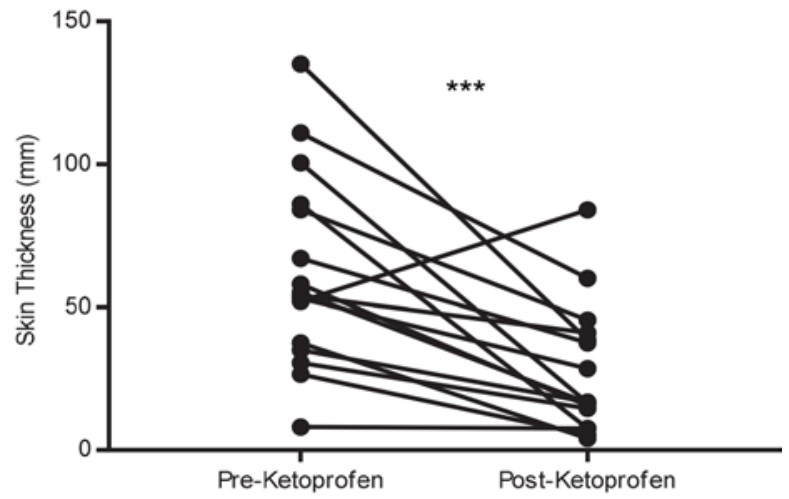

Figure 2. Ketoprofen improves cutaneous pathology and skin thickness in open-label study. (A) In the open-label trial, a 6-mm cutaneous punch biopsy was performed before and after ketoprofen therapy. The cutaneous histopathology score was calculated according to the predefined parameters, as described. Violin plot of the change $(\Delta)$ represents the value of the posttreatment minus the pretreatment score; a negative score indicates improvement. (B) For the open-label study, skin thickness was measured clinically by caliper before and after a 4-month exposure to daily ketoprofen therapy $(n=16) .\left({ }^{* * *} P<0.0001,{ }^{* *} P=0.0006\right.$, paired $t$ test $)$.

\section{Discussion}

These investigations were designed to test the hypothesis, based on preclinical experiments, that targeted antiinflammatory therapy could ameliorate the skin biology of human lymphedema, a condition for which no drug therapy is currently approved, to our knowledge. Here, we have demonstrated that treatment with ketoprofen achieved primary outcome endpoints in both the open-label and placebo-controlled exploratory studies. While it is widely acknowledged that the skin is the target organ of injury in lymphedema, clinical investigations have traditionally emphasized limb volume changes over direct assessment of the skin. Modern concepts of lymphedema pathogenesis implicate microlymphatic dysfunction within the skin; in this conceptual framework, the increase in limb volume is a consequence of this microcirculatory dysfunction. Therefore, to address the central mechanisms of lymphedema and its responsiveness to pharmacotherapy, our studies preferentially emphasize cutaneous clinical responses (skin thickness) and pathology. The improvement in skin thickness and histology in lymphedema following ketoprofen treatment is a finding that paves the way for drug therapies that will have the capacity to ameliorate lymphatic repair.

In attempting to establish the efficacy of a pharmacotherapy for lymphedema, we were faced with the problem that there were no clear antecedents upon which to base a drug trial. To begin, we initiated the open-label phase of this investigation to determine the methodology most appropriate to evaluate pharmacological treatment responses; our findings indicated that both changes in histopathology and (the more readily assessed; ref. 18, 19) skin thickness could be used as outcome measures. We therefore proceeded to a placebo-controlled confirmation of the treatment efficacy, designating skin thickness as the primary endpoint. Changes in skin thickness have previously been documented to correlate with relevant disease variables in lymphedema (20); in fact, skin thickness correlates more closely with disease severity than s.c. thickness (20).

The histopathology score was created de novo for the purposes of the current trial. The principal components of this score were dermal thickness, collagen thickness, intercellular mucin deposits, and perivascular inflammation. This empirically derived evaluation tool proved useful in both the open-label and placebo-controlled investigations. As in our previously published experience in the animal model (14), ketoprofen therapy induced therapeutic architectural remodeling of the diseased skin.

All forms of lymphedema are characterized by structural changes that include increased interstitial tissue fluid content, fibrosis of the lymphatic vasculature and surrounding tissues, adipose hypertrophy, and inflammation (12). In experimental lymphedema, there is an increase in $\mathrm{CD}^{+} \mathrm{T}$ cells, neutrophils, and macrophages (21), and DC trafficking is altered (21-23). In this study, it is noteworthy that ketoprofen treatment significantly attenuated plasma levels of G-CSF, an inflammatory cytokine known to be produced by endothelium and immune cells, including macrophages (24). While there is strong evidence 
A Placebo-Controlled Trial $1^{\circ}$ Endpoint: Skin Thickness

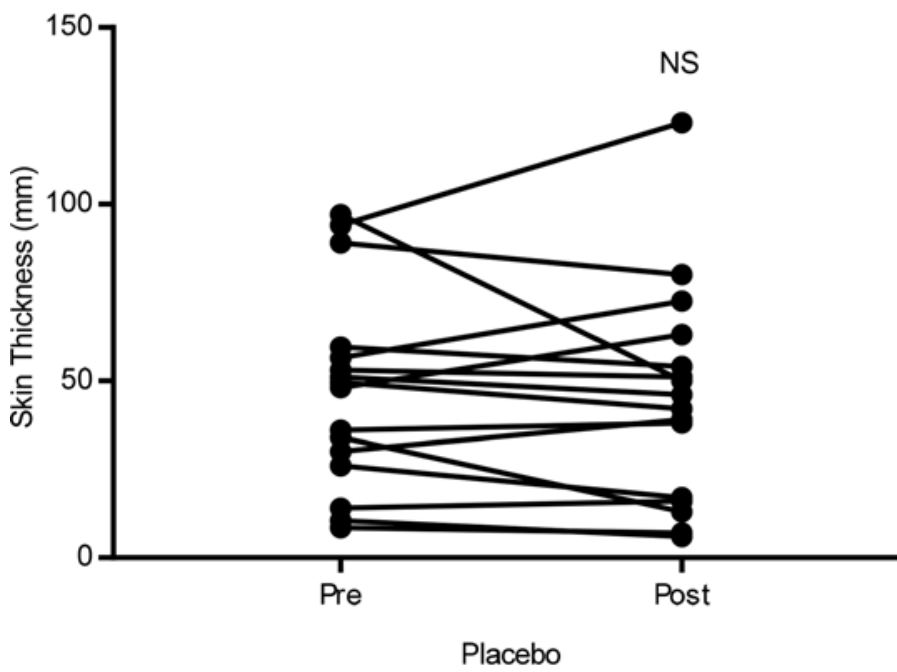

B Placebo-Controlled Trial $2^{\circ}$ Endpoint: Histology

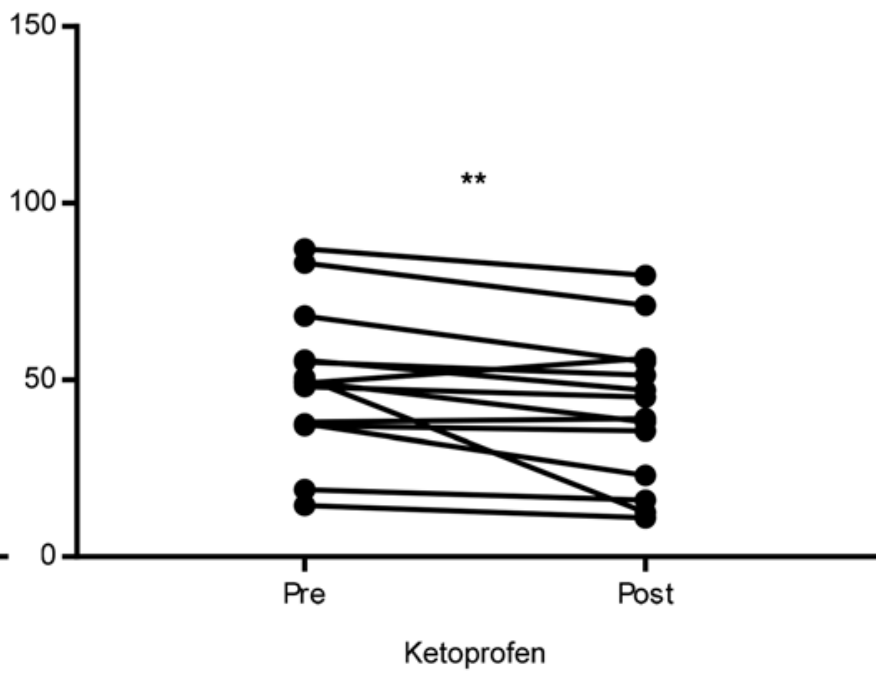

Ketoprofen

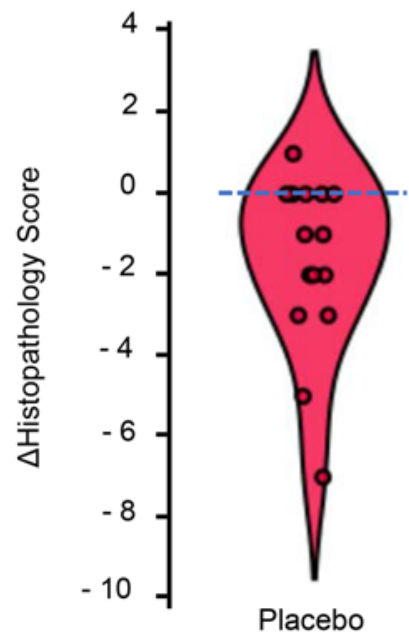

NS

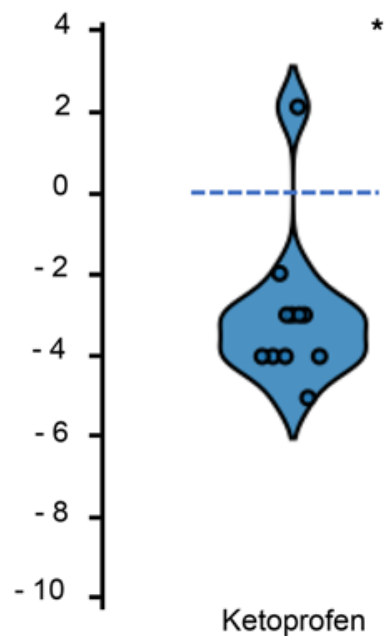

Figure 3. Ketoprofen improves skin thickness and cutaneous pathology in placebo-controlled study. (A) For the placebo-controlled trial, pre- and posttreatment skin thickness were measured for the ketoprofen $(n=14)$ and placebo groups $(n=15)$. (B) Violin plots of the changes in the blindly assessed histopathology scores. The change $(\Delta)$ represents the value of the posttreatment minus the pretreatment score. $\left({ }^{* *} P=0.01,{ }^{*} P=0.03\right.$, Mann-Whitney $U$ test.)

that macrophages and other inflammatory cells can participate in lymphatic repair (25), it is also evident that activated and polarized macrophages, including the populations that produce $\mathrm{LTB}_{4}$, can be potentially injurious to endothelium $(17,26)$. The beneficial response to ketoprofen may reflect an attenuation of these harmful innate responses.

These studies have several potentially significant limitations. Based on the studies' small sizes, we are not able to discriminate differences in the responsiveness of primary vs. secondary lymphedema. Lymphedema is a highly heterogeneous disease both in etiology and severity. Small studies are especially impacted when the starting point for enrolled patients differs greatly and reduces the power to detect clinically meaningful changes. The lack of improvement in bioimpedance or limb volume as positive secondary endpoints is unexplained, given the otherwise favorable response to ketoprofen. While improvement in bioimpedance and reduction in limb volume are intuitive measures of a lymphedema treatment effect, substantial adipose hypertrophy is responsible for most of the limb volume increase in lymphedema (27), and it is plausible that the adipocytes might not respond to the drug effect over a short, 4-month course of administration. 
Table 2. Limb volume and bioimpedance responses in the placebo-controlled trial

\begin{tabular}{lcccc}
\hline Treatment & \multicolumn{2}{c}{ limb volume $(\mathbf{m l})$} & \multicolumn{2}{c}{ Bioimpedance $\left(\mathbf{R}_{\mathbf{0}}\right)$} \\
& Pretreatment & Posttreatment & Pretreatment & Posttreatment \\
Ketoprofen & $8,598 \pm 3,066$ & $8,675 \pm 3,103^{\mathrm{A}}$ & $1.40 \pm 0.23$ & $1.39 \pm 0.24^{\mathrm{A}}$ \\
Placebo & $7,196 \pm 3,519$ & $7,256 \pm 3,496^{\mathrm{A}}$ & $1.54 \pm 0.39$ & $1.50 \pm 0.34^{\mathrm{A}}$ \\
APre-to-post comparisons not significant, paired $t$ test. & & & \\
\hline
\end{tabular}

It is also possible that these small studies were underpowered to detect small differences (i.e., a type II error). An additional confounding observation was the fact that some patients in the placebo treatment group appeared to exhibit an improved histopathology score. It is possible that assiduous lymphedema care could have provided some direct histological benefit to the placebo recipients, as has previously been observed with implementation of enhanced cutaneous hygiene (28). Finally, the result that systemic levels of G-CSF were reduced following ketoprofen treatment could be an epiphenomenon. Nevertheless, the PLS discriminant analysis (PLS-DA) discloses the interactional nature of individual changes in the measured inflammatory mediators and, thus, may be worthy of further mechanistic investigation. Despite such potential limitations, these 2 early-phase trials suggest a salutary cutaneous effect of ketoprofen for the treatment of lymphedema.

While favorable responses to ketoprofen are encouraging, the widespread, chronic use of ketoprofen is limited, as are all NSAIDs, by a black box warning regarding cardiovascular toxicity (29). This warning was issued by the US Food and Drug Administration (FDA) after our last subject was enrolled in the study. Even prior to this concern, we had continued to investigate the mechanism of ketoprofen's effect in the murine lymphedema model. We showed that the therapeutic benefit of ketoprofen was specifically attributable to its inhibition of the 5-LO metabolite, $\mathrm{LTB}_{4}(17)$. In mice, targeted $\mathrm{LTB}_{4}$ antagonism reversed edema, improved lymphatic function, and restored cutaneous architecture. In this preclinical publication, we also documented elevated $\mathrm{LTB}_{4}$ in human lymphedema sera. In vitro, $\mathrm{LTB}_{4}$ inhibited lymphangiogenesis, induced lymphatic endothelial cell apoptosis, and antagonized VEGFR3, along with Notch signaling pathways. In light of our previously published experimental data, the findings of the current study suggest that ketoprofen confers benefit in patients through its upstream inhibition of $\mathrm{LTB}_{4}$. Ubenimex, a well-tolerated oral $\mathrm{LTB}_{4}$ antagonist without anti-COX activity, had documented efficacy in the experimental model (17) and is now the subject of an international placebo-controlled trial (NCT02700529; ULTRA Trial, Eiger BioPharmaceuticals). In summary, lymphedema is a world-wide health problem that may benefit from therapies that reverse the negative impact of inflammation on lymphatic repair.

\section{Table 3. Ketoprofen reduces systemic G-CSF levels in lymphedema patients}

\begin{tabular}{|lccc|}
\hline Change in biomarker (log) & Placebo & Treated & P value \\
\hline Absolute 4 & & & $0.030 \pm 0.17$ \\
MIG & $-0.028 \pm 0.13$ & $-0.04 \pm 0.14$ & 0.39 \\
G-CSF & $0.14 \pm 0.26$ & & \\
\hline Relative 4 & & $-0.020 \pm 0.061$ & 0.041 \\
\hline G-CSF & $0.068 \pm 0.14$ & $-0.004 \pm 0.013$ & 0.072 \\
\hline IL-18 & $0.011 \pm 0.026$ & $-0.021 \pm 0.045$ & 0.080 \\
IL-17B & $0.008 \pm 0.037$ & $-0.019 \pm 0.071$ & 0.15 \\
\hline CD40 & $0.064 \pm 0.19$ & $-0.07 \pm 0.086$ & 0.082 \\
\hline EGF & $-0.004 \pm 0.11$ & $-0.020 \pm 0.035$ & 0.17 \\
\hline TRAIL & $-0.0003 \pm 0.038$ & $-0.014 \pm 0.026$ & 0.24 \\
\hline SDF1 & $-0.0003 \pm 0.032$ & $-0.011 \pm 0.009$ & 0.18 \\
\hline IL-10 & $-0.004 \pm 0.014$ & & \\
\hline
\end{tabular}

Mean change in biomarkers related to treatment of lymphedema as identified by PLS analysis. A variables importance for projection (VIP) $>1.5$ was predefined as the formal cut-off for significance. MIG, monokine induced by IFN- $\gamma$; SDF, stromal-derived factor 1. 
A Change in Total $5-\mathrm{LO}^{+}$Cells

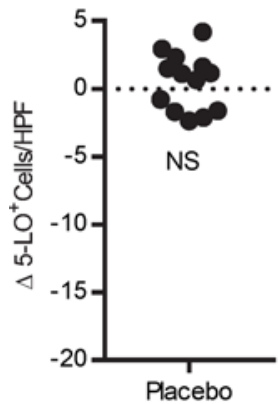

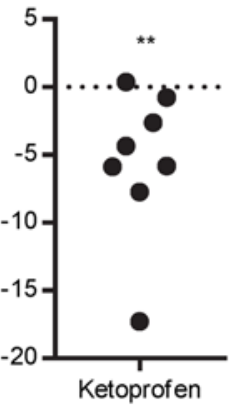

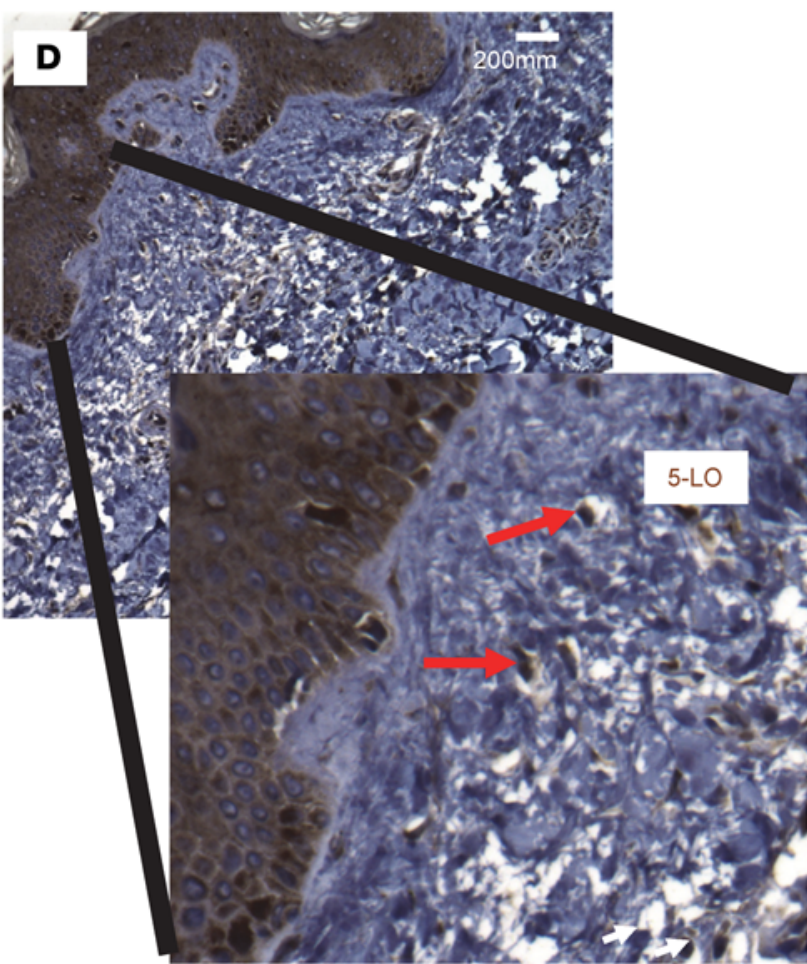

Placebo
B Change in 5-LO ${ }^{+}$Macrophages

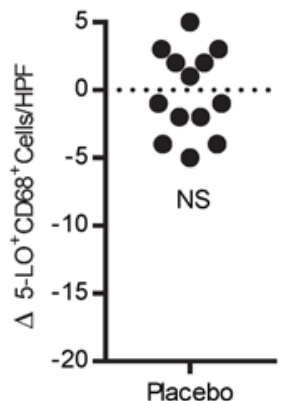

Pacebo

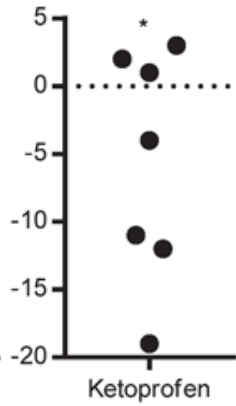

C Change in 5-LO Neutrophils

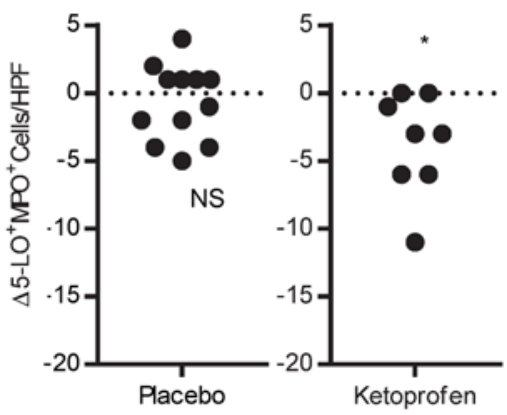

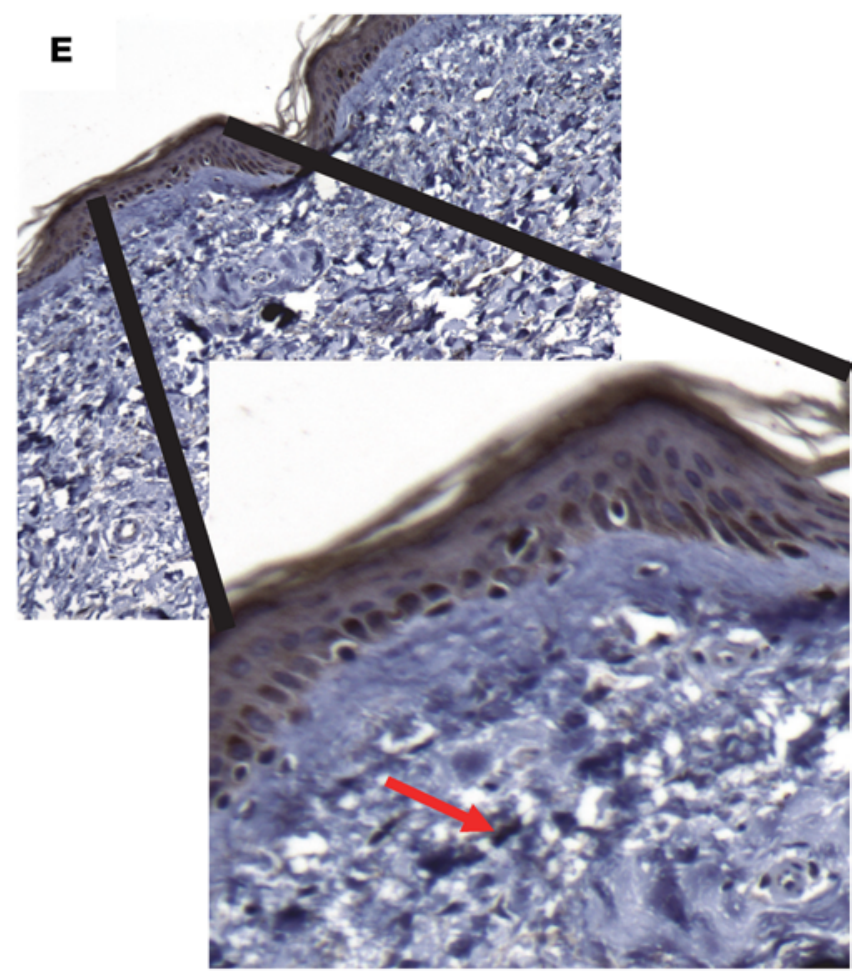

Ketoprofen

Figure 4. Ketoprofen treatment reduces cutaneous 5-LO expression. Skin biopsies obtained at the conclusion of the placebo-controlled trial were stained for 5 -LO cells (A), macrophages (B), and neutrophils (C), and cell counts were performed in a blinded manner. $\left({ }^{*} P=0.02,{ }^{* *} P=0.001\right.$, Mann-Whitney $U$ test). Representative cutaneous histology stained for 5-LO obtained at the conclusion of the trial in the placebo (D) and ketoprofen (E) groups (20x, and magnified insets). Red arrows indicate 5-LO-positive cells.

\section{Methods}

Study design, patient selection, and enrollment. This was an open-label pilot study followed by a prospective, randomized, double-blind, placebo-controlled exploratory study. Twenty-one patients were enrolled in the open-label trial, and 34 patients were enrolled in the placebo-controlled study. The primary endpoint for the open-label study was histopathological, with secondary endpoints of skin thickness, limb volume, and tissue bioimpedance changes. Guided by the results of the open-label experience, the placebo-controlled design designated skin thickness as the primary endpoint, with secondary endpoints of histopathology, limb volume, and systemic inflammatory mediators. We recruited study subjects from the patient population of the Stanford Center for Lymphatic and Venous Disorders. All patients who presented for evaluation of lymphedema between November 2010 and February 2015 ( $n=329$ ) were screened for possible enrollment. Patients with either unilateral or bilateral lymphedema of upper or lower extremities were eligible for inclusion, if the disease had a duration of $\geq 6$ months. All primary and secondary etiologies of lymphedema were eligible. Prior completion of a thorough course of decongestive physiotherapy was mandatory, as was the continuous use of all maintenance-phase physical treatment strategies, including the continuous use of properly fitted compression garments. 
Clinical and laboratory methods. Skin thickness measurements were performed with a Lange skinfold caliper (Beta Technology). For each subject, at each assessment, 3 measurements were obtained: the dorsum of the hand (or foot); the midpoint of the volar aspect of the forearm (or medial aspect of the calf); and the midpoint of the medial aspect of the upper arm (or thigh). At the initial evaluation, a dermatographic pencil was used to mark the site of each measurement. Once the locations were determined, the exact distance of each location was measured from the wrist or lateral malleolus. These locations were then reutilized for serial measurements during the trial. The calipers were calibrated prior to each use.

Limb volume was measured through serial quantitation of the limb circumference at 4-cm intervals along its long axis. The procedure was performed with gauged tape to ensure a uniform stretching force. Using these measurements, the volume of the limb was calculated with the truncated cone approximation (30). Pre- and posttreatment measurements for each subject were performed by the same operator, who, in all cases, was blinded to patient treatment status and to pretreatment measurement values at the time of the posttreatment assessment.

Bioimpedance spectroscopy was performed with the Impedimed SFB7 in all subjects. A 4-electrode configuration was used to noninvasively assess the extracellular and intracellular fluid contents of the limb (31). Data were analyzed according to Cole theory (32), using the manufacturer's software (Impedimed Ltd.), to provide values for resistance at $t=0\left(\mathrm{R}_{0}\right)$, the resistance of the extracellular fluid, including lymph; $\mathrm{R}_{\infty}$, the resistance of total tissue fluid; and $\mathrm{R}_{\mathrm{i}}$, the resistance of the intracellular fluid. For the purposes of these investigations, in all patients with unilateral lymphedema, the ratio of $\mathrm{R}_{0}$ in the affected/unaffected limbs was analyzed in each patient as a measurement of the bioimpedance attributable to the extracellular fluid content. The serial evaluations were performed by the same operator for each subject, and the operator was blinded to treatment status and to prior measurement values at the time of each assessment.

Cutaneous punch biopsy. Two contiguous 6-mm full-thickness punch biopsy specimens were obtained from the medial aspect of the forearm or calf of the affected extremity with an Acu-Punch disposable device. Biopsy specimens were immediately placed in formalin. Following biopsy, the skin edges were sutured with a single butterfly suture, and the patient received a prophylactic antibiotic regimen of cephalexin $250 \mathrm{mg}$ in 3 divided doses for each of 2 days, or an equivalent regimen. Punch biopsy was performed prior to treatment assignment and repeated on the day of final evaluation, after 4 months of continuous treatment with either ketoprofen or placebo.

Histology and IHC. Cutaneous biopsy materials were paraffin embedded and sectioned in the standard fashion. Staining with H\&E was performed on all biopsy specimens, as previously described (13). Skin samples harvested from the extremity of patients treated with ketoprofen or placebo control were embedded in paraffin and sliced in $10-\mu \mathrm{m}$-thick sections. Skin sections were subjected to immunohistochemical staining of 5-LO (Cell Signaling Technology, 3289), macrophage (Agilent Dako, KP1), or neutrophil (anti-myeloperoxidase, Ab9535, Abcam). Cutaneous lymphatic microvascular area was quantitated through immunohistochemical staining of podoplanin with anti-D2-40 (Dako, IS072). Anti-D2-40-decorated vascular structures were quantitated from digital images of the stained specimens. Nonvascular structures (glands, adipose, epidermis) were excluded.

Histology score. In the absence of an established quantitative tool, we created a numerical scoring system to serially evaluate the histopathological responsiveness of the skin to pharmacologic intervention. The dermatopathologist (J. Kim) was given a training set of 20 unblinded skin specimens, with equal numbers of lymphedema-positive and -negative cutaneous biopsy specimens. Based upon this analysis, it was determined that 4 characteristics most uniformly identified the histopathological abnormalities of untreated lymphedema: thickness of the dermal layer, intercellular mucin content, deep dermal collagen content, and perivascular infiltrate. Accordingly, the scoring system was devised to give equal weight to these 4 characteristics, each of which was assessed on a scale of increasing severity, from 0-5. The histology score for each specimen represents the arithmetic sum of the score for each of these 4 attributes. For the experimental assessments in both trials, the dermatopathologist was blinded to the treatment identity of the subjects, and histology was graded in a random order.

Phlebotomy. Blood draws were performed on all subjects in the placebo-controlled trial. The procedure was performed in the standard fashion at baseline and study conclusion, using a small-gauge needle inserted into the brachiocephalic vein. Blood $(10 \mathrm{ml})$ was withdrawn at each procedure, and the plasma was frozen at $-80^{\circ} \mathrm{C}$ for eventual biomarker analysis. 
Luminex bead assays. The assays were performed, as previously described (33), in a paired fashion on pre- and posttreatment plasma samples from each subject in the placebo-control trial. Custom human 62-plex kits were purchased from Thermo Fisher Scientific and utilized according to the manufacturer's recommendations, with modifications as described below. All of the antibodies and buffers were purchased from the manufacturer. Plasma samples were mixed with antibody-linked polystyrene beads on 96-well filter-bottom plates and incubated at room temperature for 2 hours, followed by overnight incubation at $4^{\circ} \mathrm{C}$. Plates were vacuum-filtered and washed twice with $\mathrm{PBS}+0.2 \%$ Tween-20, followed by incubation with biotinylated detection antibody for 2 hours at room temperature. Samples were then filtered and washed twice as below and resuspended in streptavidin-PE. After incubation for 40 minutes at room temperature, 2 additional vacuum washes were performed, and the samples were resuspended in reading buffer. Each sample was measured in duplicate. Plates were read using a Luminex 200 instrument with a lower bound of 100 beads per sample per analyte.

Statistics. All statistical computations utilized GraphPad Prism (Version 7.01). For normally distributed data, statistical assessments were performed with standard 2-tailed Student's $t$ test paired and unpaired comparisons, as appropriate, and significance was defined as $P<0.05$. For nonparametric comparisons, we utilized Mann-Whitney $U$ and Wilcoxon tests, as appropriate. Statistics for the Luminex assays are described above. Violin plots for noncontinuous variables made use of the visualization from BioVinci (version 1.1.3) developed by BioTuring Inc.

PLS-DA was performed using SAS software, version 9.3, and JMP Genomics, version 6.0 (SAS Institute). Samples were adjusted for age, sex, and BMI. Using PLS-DA, we assessed the relation between absolute or relative changes in circulating biomarkers and treatment assignment group (treatment or placebo). PLS-DA creates several linear combinations (latent factors) of the log-transformed predictors to maximize the covariance between the predictors and the outcome variables. We assumed that the number of latent factors was not significantly different from the model with the minimum predicted residuals sum of squares (PRESS) value. These latent variables were then used in the discrimination analysis instead of the original individual predictors (biomarkers). The importance of each cytokine in the construction of the latent variables is assessed from the VIP scores. In both continuous and categorical analysis, cytokines with a VIP $>1.5$ were considered influential. The distributions of all cytokines were analyzed by transformation to the common logarithm. The VIP score was calculated as the sum of the squared correlations between the latent factors and the biomarker, weighted for the percentage of variation explained by the latent factors in the model. Finally, volcano plots were constructed to illustrate the importance of each biomarker in the model (VIP, $x$ axis) in relation to the coefficient size of each biomarker ( $y$ axis). These volcano plots illustrate the contribution of all biomarkers to the model used to explain outcome, as well as their effect on the outcome, and are better suited for colinear correlates.

Study approval. These open-label and randomized, double-blinded placebo-controlled studies were conducted at a single academic site (Stanford University). The Administrative Panels for the Protection of Human Subjects of Stanford University (IRB 0000350) approved the protocols. Investigations were conducted according to Declaration of Helsinki principles. Written consent was obtained from all recipients prior to inclusion in the studies. The trial was registered on ClinicalTrials.gov, NCT02257970.

\section{Author contributions}

Study conception was contributed by SGR and MRN. Designed research studies were contributed by SGR, WT, and XJ. Experiments were conducted by SGR, WT, JK, JZ, BM, and JPS. Data were acquired by SGR, WT, TK, FH, BM, and LR. Data were analyzed by SGR, MRN, WT, TK, and FH. The manuscript was written by SGR, MRN, and WT.

\section{Acknowledgments}

Grant support for the authors includes The Stanford Professor of Pulmonary and Critical Care Medicine Endowed Chair Funds and Startup Funds (MRN) and the Allan and Tina Neill Endowed Chair (SGR). Luminex assays performed in the Human Immune Monitoring Center at Stanford University School of Medicine (Holden Maecker, Director).

Address correspondence to: Stanley G. Rockson, Stanford Center for Lymphatic and Venous Disorders, Falk Cardiovascular Research Center, Stanford University School of Medicine, 300 Pasteur 
Drive, Stanford, California 94305, USA. Phone: 650.725.7571; Email: rockson@stanford.edu. Or to: Mark R. Nicolls, VA Palo Alto Health Care System; Stanford University School of Medicine, Med111P, 3801 Miranda Avenue, Palo Alto, California 94304, USA. Phone: 650.723.1719; Email: mnicolls@stanford.edu.

1. Rockson SG. Update on the biology and treatment of lymphedema. Curr Treat Options Cardiovasc Med. 2012;14(2):184-192.

2. Mortimer PS, Rockson SG. New developments in clinical aspects of lymphatic disease. J Clin Invest. 2014;124(3):915-921.

3. Rockson SG. Epidemiology. In: Lee BB, Rockson SG, Bergan J eds. Lymphedema: A Concise Compendium of Theory and Practice. London: Springer; 2018:841-848.

4. Cormier JN, Askew RL, Mungovan KS, Xing Y, Ross MI, Armer JM. Lymphedema beyond breast cancer: a systematic review and meta-analysis of cancer-related secondary lymphedema. Cancer. 2010;116(22):5138-5149.

5. Rockson SG, Rivera KK. Estimating the population burden of lymphedema. Ann N Y Acad Sci. 2008;1131:147-154.

6. Rockson SG. Lymphatic Medicine: Paradoxically and Unnecessarily Ignored. Lymphat Res Biol. 2017;15(4):315-316.

7. Rutkowski JM, Moya M, Johannes J, Goldman J, Swartz MA. Secondary lymphedema in the mouse tail: Lymphatic hyperplasia, VEGF-C upregulation, and the protective role of MMP-9. Microvasc Res. 2006;72(3):161-171.

8. Zampell JC, et al. Lymphatic function is regulated by a coordinated expression of lymphangiogenic and anti-lymphangiogenic cytokines. Am J Physiol, Cell Physiol. 2012;302(2):C392-C404.

9. Jin DP, An A, Liu J, Nakamura K, Rockson SG. Therapeutic responses to exogenous VEGF-C administration in experimental lymphedema: immunohistochemical and molecular characterization. Lymphat Res Biol. 2009;7(1):47-57.

10. Szuba A, et al. Therapeutic lymphangiogenesis with human recombinant VEGF-C. FASEB J. 2002;16(14):1985-1987.

11. Kim H, Kataru RP, Koh GY. Inflammation-associated lymphangiogenesis: a double-edged sword? J Clin Invest. 2014;124(3):936-942.

12. Jiang X, Nicolls MR, Tian W, Rockson SG. Lymphatic Dysfunction, Leukotrienes, and Lymphedema. Annu Rev Physiol. 2018;80:49-70.

13. Tabibiazar R, et al. Inflammatory manifestations of experimental lymphatic insufficiency. PLoS Med. 2006;3(7):e254.

14. Nakamura K, Radhakrishnan K, Wong YM, Rockson SG. Anti-inflammatory pharmacotherapy with ketoprofen ameliorates experimental lymphatic vascular insufficiency in mice. PLoS One. 2009;4(12):e8380.

15. Ambrus JL, Haneiwich S, Chesky L, McFarland P, Engler RJ. Improved in vitro antigen-specific antibody synthesis in two patients with common variable immunodeficiency taking an oral cyclooxygenase and lipoxygenase inhibitor (ketoprofen). J Allergy Clin Immunol. 1991;88(5):775-783.

16. Rajić Z, Hadjipavlou-Litina D, Pontiki E, Kralj M, Suman L, Zorc B. The novel ketoprofen amides--synthesis and biological evaluation as antioxidants, lipoxygenase inhibitors and cytostatic agents. Chem Biol Drug Des. 2010;75(6):641-652.

17. Tian W, et al. Leukotriene B4 antagonism ameliorates experimental lymphedema. Sci Transl Med. 2017;9(389):eaal3920.

18. Roberts CC, Levick JR, Stanton AW, Mortimer PS. Assessment of truncal edema following breast cancer treatment using modified Harpenden skinfold calipers. Lymphology. 1995;28(2):78-88.

19. Williams AF, Vadgama A, Franks PJ, Mortimer PS. A randomized controlled crossover study of manual lymphatic drainage therapy in women with breast cancer-related lymphoedema. Eur J Cancer Care (Engl). 2002;11(4):254-261.

20. Mellor RH, Bush NL, Stanton AW, Bamber JC, Levick JR, Mortimer PS. Dual-frequency ultrasound examination of skin and subcutis thickness in breast cancer-related lymphedema. Breast J. 2004;10(6):496-503.

21. Zampell JC, Yan A, Elhadad S, Avraham T, Weitman E, Mehrara BJ. CD4(+) cells regulate fibrosis and lymphangiogenesis in response to lymphatic fluid stasis. PLoS ONE. 2012;7(11):e49940.

22. Ly CL, Kataru RP, Mehrara BJ. Inflammatory Manifestations of Lymphedema. Int J Mol Sci. 2017;18(1):E171.

23. Weitman ES, et al. Obesity impairs lymphatic fluid transport and dendritic cell migration to lymph nodes. PLoS One. 2013;8(8):e70703.

24. Panopoulos AD, Watowich SS. Granulocyte colony-stimulating factor: molecular mechanisms of action during steady state and 'emergency' hematopoiesis. Cytokine. 2008;42(3):277-288.

25. Kerjaschki D. The lymphatic vasculature revisited. J Clin Invest. 2014;124(3):874-877.

26. Tian W, et al. Blocking macrophage leukotriene b4 prevents endothelial injury and reverses pulmonary hypertension. Sci Transl Med. 2013;5(200):200ra117.

27. Brorson H, Ohlin K, Olsson G, Nilsson M. Adipose tissue dominates chronic arm lymphedema following breast cancer: an analysis using volume rendered CT images. Lymphat Res Biol. 2006;4(4):199-210.

28. Stocks ME, Freeman MC, Addiss DG. The Effect of Hygiene-Based Lymphedema Management in Lymphatic Filariasis-Endemic Areas: A Systematic Review and Meta-analysis. PLoS Negl Trop Dis. 2015;9(10):e0004171.

29. [No authors listed]. FDA Drug Safety Communication: FDA strengthens warning that non-aspirin non-steroidal anti-inflammatory drugs (NSAIDS) can cause heart attacks and strokes. Food and Drug Administration. https://www.fda.gov/Drugs/ DrugSafety/ucm451800.htm. Updated Feburary 26, 2018. Accessed September 18, 2018.

30. Sitzia J. Volume measurement in lymphoedema treatment: examination of formulae. Eur J Cancer Care (Engl). 1995;4(1):11-16.

31. Ward LC, Dylke E, Czerniec S, Isenring E, Kilbreath SL. Confirmation of the reference impedance ratios used for assessment of breast cancer-related lymphedema by bioelectrical impedance spectroscopy. Lymphat Res Biol. 2011;9(1):47-51.

32. Cornish BH, Thomas BJ, Ward LC. Improved prediction of extracellular and total body water using impedance loci generated by multiple frequency bioelectrical impedance analysis. Phys Med Biol. 1993;38(3):337-346.

33. Lin S, et al. Prospective transcriptomic pathway analysis of human lymphatic vascular insufficiency: identification and validation of a circulating biomarker panel. PLoS ONE. 2012;7(12):e52021. 